\title{
Research on Analysis Method of Traffic Congestion Mechanism Based on Improved Cell Transmission Model
}

\section{Hongzhao Dong, Shuai Ma, Mingfei Guo, and Dongxu Liu}

The Joint Institute of Intelligent Transportation System, Zhejiang University of Technology, Hangzhou 310014, China

Correspondence should be addressed to Hongzhao Dong, its@zjut.edu.cn

Received 8 August 2012; Accepted 21 October 2012

Academic Editor: Wuhong Wang

Copyright (c) 2012 Hongzhao Dong et al. This is an open access article distributed under the Creative Commons Attribution License, which permits unrestricted use, distribution, and reproduction in any medium, provided the original work is properly cited.

To analyze the spreading regularity of the initial traffic congestion, the improved cell transmission model (CTM) is proposed to describe the evolution mechanism of traffic congestion in regional road grid. Ordinary cells and oriented cells are applied to render the crowd roads and their adjacent roads. Therefore the traffic flow could be simulated by these cells. Resorting to the proposed model, the duration of the initial traffic congestion could be predicted and the subsequent secondary congestion could be located. Accordingly, the spatial diffusion of traffic congestion could be estimated. At last, taking a road network region of Hangzhou city as an example, the simulation experiment is implemented to verify the proposed method by PARAMICS software. The result shows that the method could predict the duration of the initial congestion and estimate its spatial diffusion accurately.

\section{Introduction}

The urban traffic congestion has been becoming a more and more serious issue, especially in China. Traffic congestion is a typical traffic condition with a dynamic course by time. The congestion generating mechanisms analysis considering both time and space scale helps to illustrate the dynamic changing characteristic of the traffic flow that eventually leads to the congestion. Many scholars had studied the urban traffic congestion by cell transmission model (CTM) at the 1990s [1-3]. Some scholars, such as Szeto, Jia Bin et al., had researched CTM model to have a good performance in simulating the typical dynamic characteristics of traffic flow, such as the formation of shock waves, traffic congestion, and the dynamic evolution rule of node combined by Multiroad $[4,5]$. Certainly, other scholars, such as Chen Qian, ZHOU Xi-peng, and YANG Zhao-shen, applied CTM model to the models of traffic 
flow spread on network such as traffic bottleneck recognition and modeling for traffic events duration time and traffic recovery time [6-10].

In previous studies, the spatial diffusion estimating and duration predicting of traffic congestion are separately supposed as both independent issues on congestion. However, both issues usually affect and restrain one another. Actually they have remarkable relationship in time and space scale. Therefore, the analysis method based on our proposed improved CTM model will give a new solution to track traffic congestion considering both time and space scale. And the research in this paper promises to prevent and relieve traffic congestion and improve the utilization efficiency of the road resources.

This paper is organized as follows. The spatial diffusion regularity of traffic congestion is analyzed in Section 2. The improved cell transmission model is given to describe the spread of traffic flow in the road grid in Section 3. The analysis method of traffic congestion mechanism based on improved CTM is researched in Section 4. In Section 5, taking a road network region of Hangzhou city as an example, the experimental simulation is provided to demonstrate the application of our proposed method by PARAMICS software. The last section highlights the conclusion.

\section{Spatial Diffusion Analysis of Traffic Congestion}

Traffic congestion refers to traffic flow detained phenomenon caused by the contradiction between traffic requirement and traffic capacity. Generally, traffic congestion would occur if the traffic capacity supplied by traffic facilities is close to or less than the current traffic demand. In some specific time, the regular bottlenecks will be formed if the traffic demand keeps being beyond the transportation capacity. Such case is called regular traffic congestion. Besides, if the actual capacity declines because of temporary event or accident, occasional traffic congestion is generated.

For a jammed unit in the road network, if the traffic demands remain high level and even continue to increase, the actual traffic capacity to collect the upstream traffic flow will become more and more limited. It leads to slow traffic speed and quickly rising traffic density of the upstream unit. That is how the primary traffic congestion can spread to upstream unit. In addition, traffic flow is usually controlled by traffic signal control system. Consequently, traffic flow in jammed unit with small headway will spread to the downstream unit during the green time. Traffic demand and $v / c$ of downstream unit will rise rapidly. With the increasing interference between the vehicles with one another, the traffic speed will be dropped and it may also lead to the possible congestion. These factors easily make the traffic flow unstable and the capacity to drop. It is why the downstream units may be jammed. Therefore, the original congestion can spread to the whole road grid quickly. If there are no effective corresponding measures to relieve its spatial diffusion, the whole or regional traffic congestion will occur.

\section{Improved Cell Transmission Model}

\subsection{Cell Transmission Model}

Cell transmission model (CTM) is based on assumption that the traffic flow $q$ and density $\rho$ have a relationship as shown in Figure 1. 


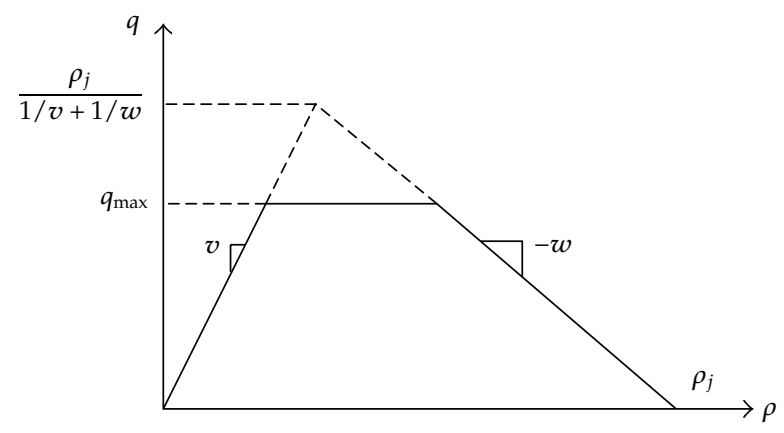

Figure 1: A trapezoidal fundamental diagram for the cell transmission model.

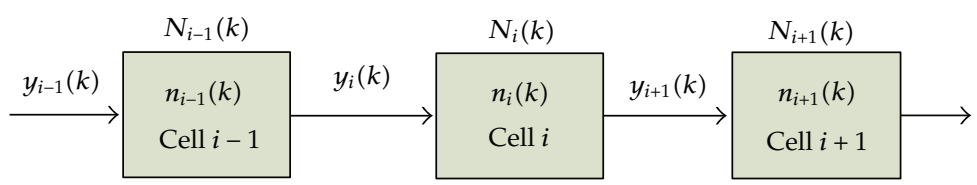

Figure 2: Traffic flow moving model of CTM.

CTM means the road section could be divided into several cells and time series discredited into several time steps. The length of each cell is the driving distance with free traffic flow during one time step. It should satisfy the below formula to describe the traffic flow between two nearby cells by CTM:

$$
q=\min \left\{v \rho, q_{\max }, w\left(\rho_{j}-\rho\right)\right\}
$$

Here $v$ is free traffic flow speed and $w$ is back propagation speed of traffic wave when it is jammed. $q_{\max }$ is maximum traffic volume. Under low traffic density, $v$ is constant. While traffic density is high, $w$ is constant and $v>w$. For $0<\rho<\rho_{j}$, the above quantity is satisfied.

The traffic flow spreading process between cells is shown as Figure 2. At time step $k$, $y_{i}(k)$ is the total flow entering the cell $i . N_{i}(k)$ is the maximum capacity of the cell $i . n_{i}(k)$ is the number of vehicles in the cell $i$.

$q_{i}(k)$ is the inflow rate of the cell $i$ and $q_{i \cdot \max }(k)$ is the maximum inflow rate of the cell $i$. And the function relation between vehicles and the density of cell is as the following equation:

$$
n_{i}(k)=\rho_{i}(k) v \delta
$$

So it is deduced the traffic flow spread relationship between two adjacent cells is as (3.3). $Q_{i}(k)$ is the maximum inflow rate of cell $i$. Then after discretizing it, traffic flow conservation equation in CTM is as (3.4):

$$
\begin{aligned}
y_{i}(k)= & \min \left\{n_{i-1}(k), Q_{i}(k), \frac{\omega\left(N_{i}(k)-n_{i}(k)\right)}{v}\right\}, \\
& n_{i}(t+1)=n_{i}(t)+y_{i}(t)-y_{i+1}(t) .
\end{aligned}
$$




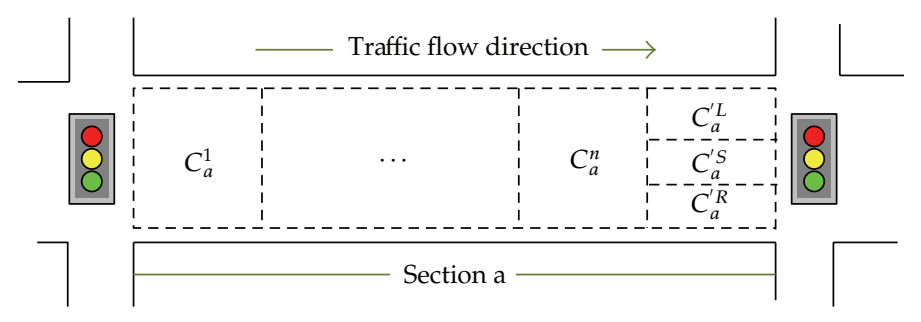

Figure 3: Cell definition improved CTM.

The existing CTM method is appropriated for describing traffic flow of linear road such as freeway. Unfortunately, the weakness of node diversion and node confluence appears in the existing CTM and it is unable to suit for traffic flow modeling of urban road network. Accordingly, the improved CTM method is researched as below.

\subsection{Improved CTM and Its Cell Definition}

The spread of traffic flow in the road grid could be classed into two aspects. One is traffic flow transmission inside the road section and another is between the adjacent sections. Recently many scholars use Daganzo's cell transmission model to construct the spread of traffic flow which includes section model and node model. The node model involves the signal control and oriented lanes at the end of the section. Based on the node model, the improved section model is proposed with oriented cell instead of node model into section model to realize the spread of traffic flow. It helps to simplify the modeling process and improve the calculation efficiency.

Oriented cell is defined as the cell locating at oriented lane of section. It plays the role of traffic flow diverging. The capacity and outflow of oriented cells is decided by the timing scheme of the downstream traffic control system. Therefore the key parameters of oriented cell are different because of their different traffic flow direction.

According to the principle of lane group classification, define $C_{k}^{L}, C_{k}^{S}$, and $C_{k}^{R}$ as three kinds of oriented cells to describe spatial distribution of traffic flow shown as Figure 3 . It is essential to analyze the spatial diffusion of traffic congestion.

\subsection{Parameter Definition and Constraint Condition}

Along the traffic direction, section $a$ consists of ordinary cells $C_{a}^{i}(i=1,2, \ldots, m \cdot i$ is numbered in ascending order from 1 along the traffic direction.) and a series of oriented cells. At time step $k$, parameter of two types of cell is shown as in Table 1.

According to the core theory of CTM, traffic flow transmission between cells could be classified in the three types below.

The first type is the flow expression of ordinary cells in the same section shown as in (3.3).

The second type is the flow expression of ordinary cell and oriented cells in the same section shown in (3.5), (3.6), and (3.7). This type describes that the traffic flow 
Table 1: Parameter definition of model.

\begin{tabular}{lcc}
\hline Parameter & Ordinary cell & Oriented cell \\
\hline Density & $\rho_{a}^{i}(k)$ & $\rho_{a}^{\xi}(k)$ \\
Vehicles & $n_{a}^{i}(k)$ & $n_{a}^{\xi}(k)$ \\
Inflow rate & $q_{a}^{i}(k)$ & $q_{a}^{\xi}(k)$ \\
Inflow & $y_{a}^{i}(k)$ & $y_{a}^{\xi}(k)$ \\
Max. outflow & $q_{a}^{i, \max }(k)$ & $q_{a}^{\xi, \max }(k)$ \\
Max. inflow & $Q_{a}^{i}(k)$ & $Q_{a}^{\xi}(k)$ \\
Capacity & $N_{a}^{i}(k)$ & $N_{a}^{\xi}(k)$ \\
\hline
\end{tabular}

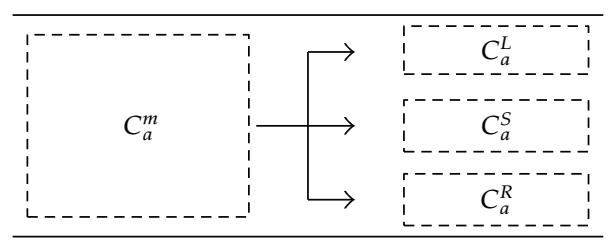

Figure 4: Flow transmission between ordinary cell and oriented cells.

of the ordinary cell moves into different oriented cells on the basis of trip demand shown in Figure 4:

$$
\begin{gathered}
y_{a}^{L}(k)=\min \left\{n_{a}^{m}(k) \eta_{L}, Q_{a}^{L}(k), \frac{\omega\left[N_{a}^{L}(k)-n_{a}^{L}(k)\right]}{v}\right\}, \\
y_{a}^{R}(k)=\min \left\{n_{a}^{m}(k) \eta_{R}, Q_{a}^{R}(k), \frac{\omega\left[N_{a}^{R}(k)-n_{a}^{R}(k)\right]}{v}\right\}, \\
y_{a}^{S}(k)=\min \left\{n_{m}(k)\left(1-\eta_{L}-\eta_{R}\right), Q_{a}^{S}(k), \frac{\omega\left[N_{a}^{S}(k)-n_{a}^{S}(k)\right]}{v}\right\},
\end{gathered}
$$

$\eta_{L}, \eta_{R}$ are the behavior proportion of left turn and right turn of traffic flow in the section.

The third type is the flow expression of oriented cell and ordinary cells of nearby sections shown in (3.8). This type describes that the traffic flow of the oriented cell moves into ordinary cells of different downstream sections shown in Figure 5:

$$
Q_{a}^{\xi}(k)=\min \left\{\frac{g_{a}^{\xi}(k)}{h(v)}, \frac{w\left[N_{a}^{\xi}(k)-n_{a}^{\xi}(k)\right]}{v}, q_{a}^{\xi, \max } \delta\right\},
$$

$\xi$ can express $L, S$, or $R . g_{a}^{\xi}(k)$ is green time in the direction of traffic flow in oriented cell by signal control system. $h(v)$ is the average headway of free traffic flow. 


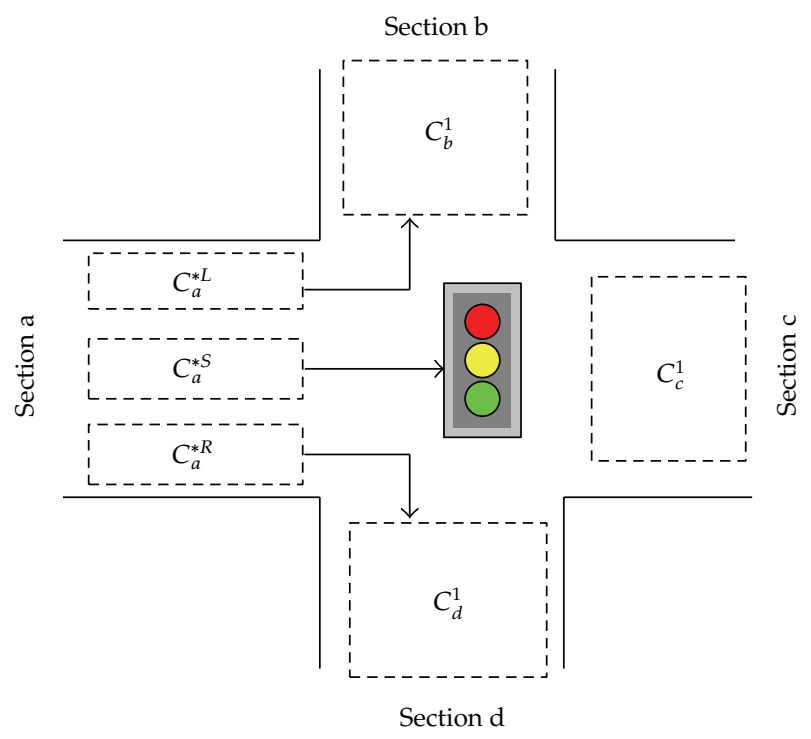

Figure 5: Flow transmission between oriented cells and ordinary cells.

Therefore flow expression is updated in (3.9):

$$
y_{b}^{1}(k)=\min \left\{n_{b}^{1}(k), Q_{a}^{\xi}(k)\right\}
$$

If the model is applied to urban road grid, the oriented cell and the ordinary cell is difficult to be defined as equal length. It will lead to the strong noise of model. The traffic density conservation function is introduced to solve the issue. The function can ignore the cell length inequality that leads to disorder of traffic flow transmission. The general formula is shown in (3.10):

$$
\rho_{i}(k+1)=\rho_{i}(k)+\frac{\delta}{l_{i}}\left(y_{i}(k)-y_{i+1}(k)\right) .
$$

Traffic density conservation formula for the second and third type is shown in (3.11):

$$
\frac{\sum_{\xi=R, S, L} \rho_{a}^{\xi}(k+1)}{3}=\frac{\sum_{\xi=R, S, L} \rho_{a}^{\xi}(k)}{3}+\frac{\delta}{l_{a}^{\delta}}\left[y_{a}^{m}(k)-y_{b}^{1}(k)\right] .
$$

\section{Analysis Method for Traffic Congestion Mechanism Based on Improved CTM}

The analysis method for traffic congestion mechanism contains both of predicting and locating, that is, to predict the duration of initial congestion and to locate secondary congestion. To improve the accuracy of the method, the sections can be divided into three groups according to their locations and the traffic flow direction. The first one is the object group that is being 


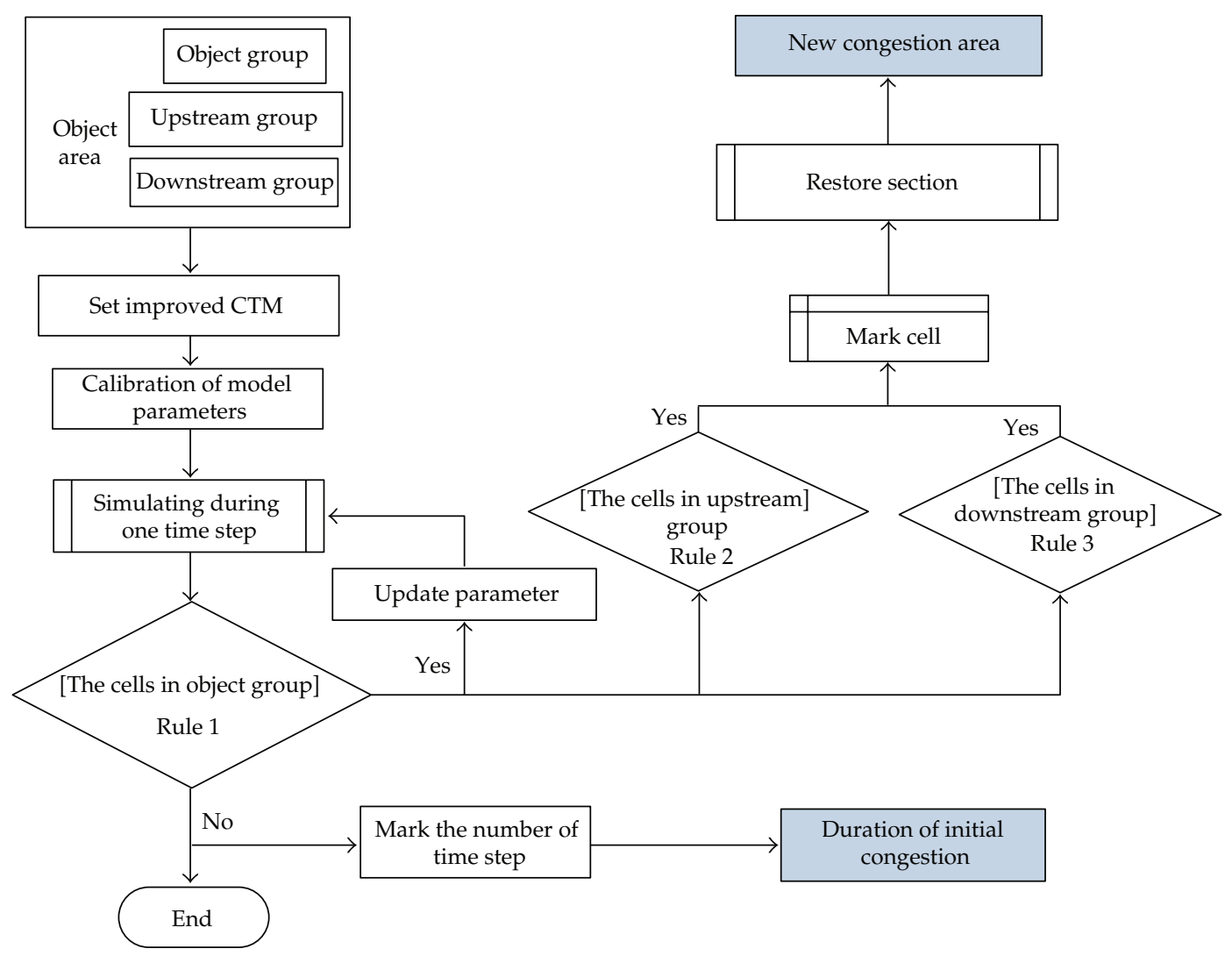

Figure 6: Flowchart of analysis method for traffic congestion mechanism based on CTM.

jammed sections. The second group is the upstream group including the sections locating at upstream of the object group. The third group is the downstream group that locates downstream of object section.

The analysis method for traffic congestion mechanism based on CTM is as follows. The analytical cycle is discretized into several time steps. The dynamic traffic characteristic is simulated by the inflow vehicles and outflow vehicles during the period of one time step. After every time step is completed, it will repeatedly output the value of the key variables such as the inflow vehicle quantity and the traffic capacity of the section until the termination conditions are met. After each step, the cells in different groups are judged by the corresponding rules. If the cell complies with the rules, it will be marked until the end condition. The marked cells will restore the section. The outcome of new congestion area and duration of initial congestion can be obtained eventually. The flow chart of the method is shown in Figure 6.

All cells in object group are analyzed to predict the duration of the initial congestion. If all cells appear to be noncongestion status after $\lambda$ time steps, the duration of initial congestion is as (4.1):

$$
T_{\text {last }}=\lambda \delta \text {. }
$$


The ordinary cells in the second group and the third group are analyzed to locate the secondary congestion. After repeatedly simulating and comparing with the practice data, the judgment rules are introduced to indentify traffic state of cell. Rule 1 describes the judgment rule of the cell of section $p$ in the object group.

Rule 1. If there is $y_{p}^{i+1}(k)=n_{p}^{i}(k)$, then $C_{p}^{i}(k) \notin U_{c g t}(k)$. Otherwise $C_{p}^{i}(k) \in U_{c g t}(k)$.

$U_{c g t}(k)$ is the set of congestion cells after No. $k$ time step. When $U_{c g t}(\lambda)$ is empty, it means the initial congestion ends.

Rules 2 and 3 describe the judgment rules of the cell of section $a$ in the upstream group and the cell of section $b$ in the upstream group during the period $T_{\gamma}$ which is the dura-tion of initial congestion.

Rule 2. If there is $f\left(T_{\gamma}\right)=\operatorname{count}\left[y_{a}^{i}(k)<n_{a}^{i}(k)\right] \geq \varepsilon_{\exp 1}$, then $C_{a}^{i}(k) \in U_{c g t}\left(T_{\gamma}\right)$. Otherwise $C_{a}^{i}(k) \notin U_{c g t}\left(T_{\gamma}\right)$.

Rule 3. If there is $f\left(T_{\gamma}\right)=\operatorname{count}\left\{y_{b}^{i}(k)=\left[N_{b}^{i}(k)-n_{b}^{i}(k)\right]\right\} \geq \varepsilon_{\exp 2}$, then $C_{b}^{i}(k) \in U_{c g t i}\left(T_{\gamma}\right)$. Otherwise $C_{a}^{i}(k) \notin U_{c g t}\left(T_{\gamma}\right)$.

$f\left(T_{\gamma}\right)=$ count(expression) means the number of variables of cell satisfy the expression after each time step. $\varepsilon_{\text {exp }}$ is the default threshold.

Above all, the analysis method for traffic mechanism based on CTM can be divided into four steps.

Step 1. Define the cells of three groups and assign values to basic variables.

Step 2. Initialize the numbers of vehicles, the capacity, and other variables of each cell.

Step 3. The vehicles transmit between cells referring to (3.5), (3.6), (3.7), and (3.9). Cells are divided into sets according to Rules 1-3 after each time step.

Step 4. Repeat Step 3 until satisfying the terminating condition.

\section{Simulation Experiment of Our Proposed Method by PARAMICS}

PARAMICS is the reliable, feature-rich microscopic traffic simulation software, which is widely applied to research intelligent transportation. The simulation experiment contains road network modeling supported by modeller module and API programming supported by programmer module.

The road network model has been constructed referring to the location and spatial relationship of real road network in Hangzhou. The traffic control system of each node in network is set by the typical actual signal timing plan. The traffic detector is fixed at the beginning of each cell to virtually realize the cell definition in the model shown in Figure 7. The traffic flow acquired by the detector can promise to verify the validity and feasibility of the proposed method.

Here are some parameters in the experiment. The congestion density of each lane is $142 \mathrm{puc} / \mathrm{km}$. The free-flow speed is $36 \mathrm{~km} / \mathrm{h}$. Time step is $10 \mathrm{~s}$. The length of ordinary cell is $100 \mathrm{~m}$. The capacity of each lane in ordinary cell is 14 puc. Assuming at the beginning the section of Tiyuchang Road from Wulin Crossing to Yan'an Crossing is jammed and the traffic congestion is triggered by short-term traffic demand expansion. 


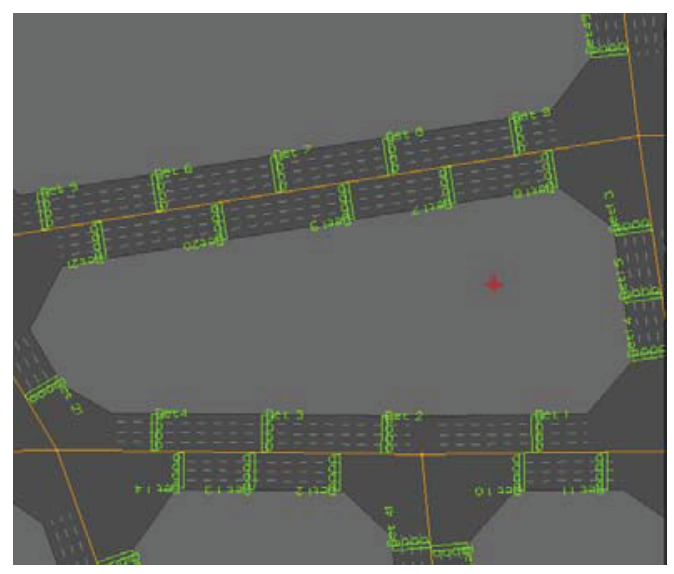

Figure 7: Virtual cell of the network.

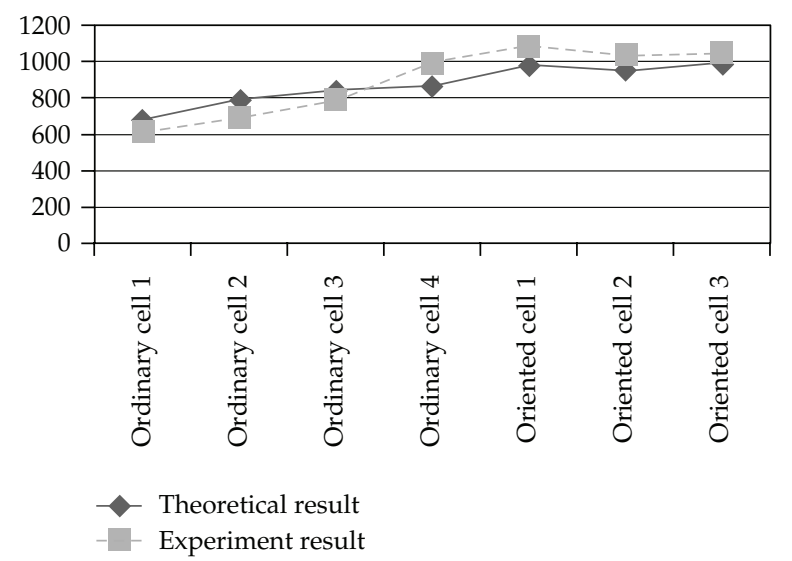

Figure 8: Comparing experiment and theoretical results.

The experiment results of duration of initial congestion are compared with the theoretical value shown in Figure 8.

The density of each ordinary cell in object group is shown in Figure 9. The above curves shows that cell1, cell2, cell3, and cell4 separately have obvious downward trend during [600s-720s], [720s-840s], [840s-960s], [960s-1080s]. Both of the duration of initial conges-tion in the experiment and its theoretical result locates in these areas.

The above results demonstrate that the max duration of congestion is $930 \mathrm{~s}$ of all cells in the object group. Therefore during 96 time steps, the traffic state of sections of upstream and downstream groups is influenced by initial congestion shown in Table 2.

The above table suggests that the influence to upstream section by initial congestion is more than one to downstream section. Vehicles queuing phenomenon is the most significant characteristic of congestion. The vehicles from upstream join the queue and the queue is lengthened into upstream section. Therefore, the congestion spreads to upstream sections. Besides, the vehicle queue is intermittently starting and stopping because of traffic control system. The influence to downstream section is related to the signal time plan by traffic control system. What is more, the improved CTM have good performance on modeling traffic 
Table 2: Results of second congestion location.

\begin{tabular}{|c|c|c|c|c|c|c|}
\hline \multirow[t]{2}{*}{ Time step } & \multicolumn{2}{|c|}{$\begin{array}{l}\text { The number of } \\
\text { congestion cells in } \\
\text { upstream group }\end{array}$} & \multicolumn{2}{|c|}{$\begin{array}{c}\text { The number of } \\
\text { congestion cells in } \\
\text { downstream group }\end{array}$} & \multicolumn{2}{|c|}{ Precision } \\
\hline & $\begin{array}{c}\text { Theoretical } \\
\text { result }\end{array}$ & $\begin{array}{l}\text { Experiment } \\
\text { result }\end{array}$ & $\begin{array}{c}\text { Theoretical } \\
\text { result }\end{array}$ & $\begin{array}{l}\text { Experiment } \\
\text { result }\end{array}$ & $\begin{array}{l}\text { Matching } \\
\text { number }\end{array}$ & $\begin{array}{l}\text { Precision } \\
\text { rate }\end{array}$ \\
\hline $1-12$ & 3 & 3 & 1 & 0 & 2 & $91.67 \%$ \\
\hline $13-24$ & 5 & 5 & 2 & 2 & 4 & $83.33 \%$ \\
\hline $25-36$ & 6 & 7 & 2 & 3 & 6 & $83.33 \%$ \\
\hline $37-48$ & 6 & 6 & 3 & 3 & 7 & $87.5 \%$ \\
\hline $49-60$ & 5 & 6 & 3 & 3 & 6 & $83.33 \%$ \\
\hline 61-72 & 5 & 4 & 2 & 2 & 5 & $91.67 \%$ \\
\hline $73-84$ & 4 & 3 & 2 & 1 & 4 & $91.67 \%$ \\
\hline $85-96$ & 3 & 2 & 1 & 1 & 2 & $87.5 \%$ \\
\hline
\end{tabular}

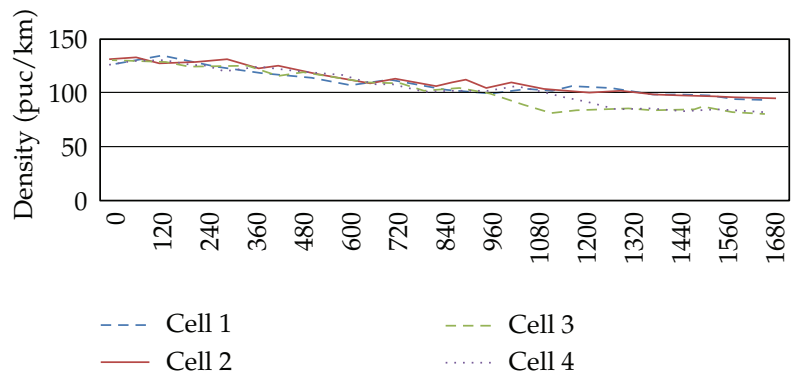

Figure 9: Density curves of ordinary cells in object group.

flow of the urban road grid and describing the congestion issue considering time and space scales at the same time comparing the CTM.

\section{Conclusion}

The improved cell transmission model with the oriented cell instead of node model is proposed to realize the spreading of traffic flow in the road grid, which could not only simplify the model but also improve the adaptability of the model. The analysis method of traffic congestion mechanism based on the model could be applied to predict the duration of the initial congestion and locate the secondary congestion. Besides, the micro-simulation experiments demonstrate the validity and feasibility of our proposed comprehensive method, which can satisfy the analytical requirements of traffic congestion in the urban transportation.

\section{Acknowledgment}

The research is support by National Natural Science Foundation of China (Grant no. 61174176). 


\section{References}

[1] C. F. Daganzon, "The lagged cell transmission model," in Transportation an Traffic Theory, A. Ceder, Ed., pp. 81-103, Ergamon-Elsevier, New York, NY, USA, 1999.

[2] C. F. Daganzo, "The cell transmission model: a dynamic representation of highway traffic consistent with the hydrodynamic theory," Transportation Research Part B, vol. 28, no. 4, pp. 269-287, 1994.

[3] W. Y. Szeto, "Enhanced lagged cell-transmission model for dynamic traffic assignment," Transportation Research Record, no. 2085, pp. 76-85, 2008.

[4] L. Muñoz, X. Sun, R. Horowitz, and L. Alvarez, "Piecewise-linearized cell transmission model and parameter calibration methodology," Transportation Research Record, no. 1965, pp. 183-191, 2006.

[5] B. Jia, Z. Y. Gao, K. P. Li, and X. G. Li, Models and Simulations of Traffic System Based on Theory of Cellular Automaton, Science Press, Beijing, China, 2007.

[6] Z. S. Yang, X. Y. Gao, and D. Sun, "Cellular automata model of urban traffic emergency evacuation and rescue," Journal of Traffic and Transportation Engineering, vol. 11, no. 2, pp. 114-120, 2011.

[7] J. C. Long, Studies on Congestion Propagation Properties and Dissipation Control Strategies of Urban Road Traffic, Beijing Jiaotong university, 2009.

[8] Q. Chen and W. Wang, "Application of fuzzy optimization method based on CTM for traffic trunk line control under special events," Journal of Southeast University (Natural Science Edition), vol. 38, no. 5, pp. 861-865, 2008.

[9] B. S. Kerner, H. Rehborn, M. Aleksic, and A. Haug, "Recognition and tracking of spatial-temporal congested traffic patterns on freeways," Transportation Research Part C, vol. 12, no. 5, pp. 369-400, 2004.

[10] A. P. Lian, Z. Y. Gao, and J. C. Long, “Dynamic user optimal assignment problem of link variables based on the cell transmission model," Acta Automatica Sinica, vol. 33, no. 8, pp. 852-859, 2007. 


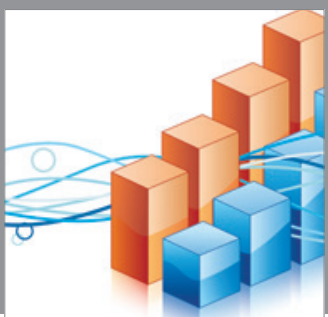

Advances in

Operations Research

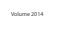

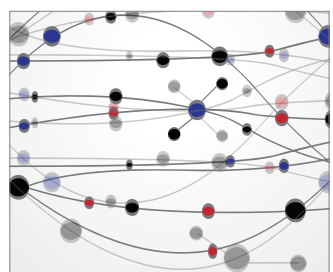

\section{The Scientific} World Journal
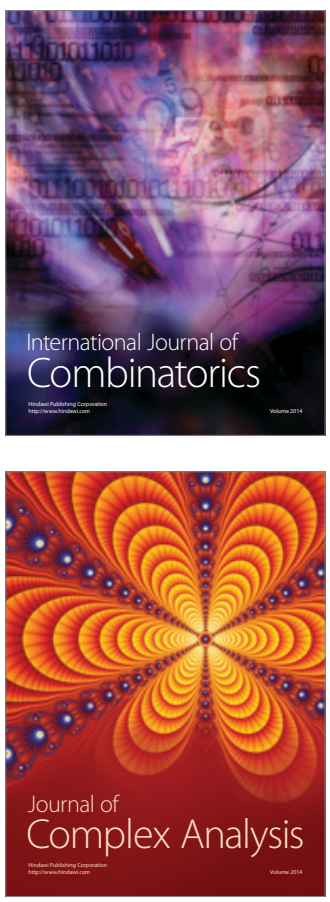

International Journal of

Mathematics and

Mathematical

Sciences
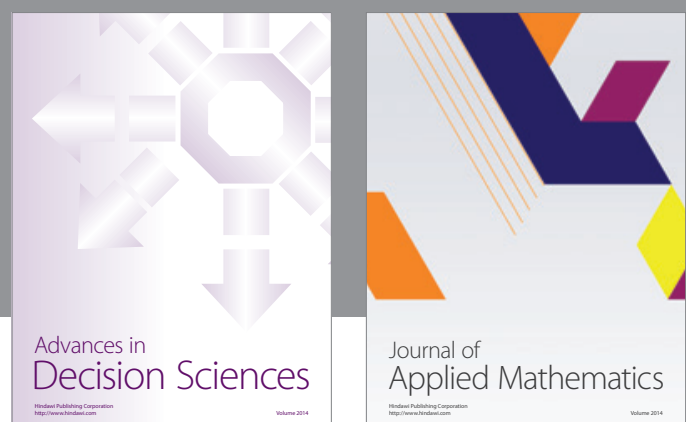

Journal of

Applied Mathematics
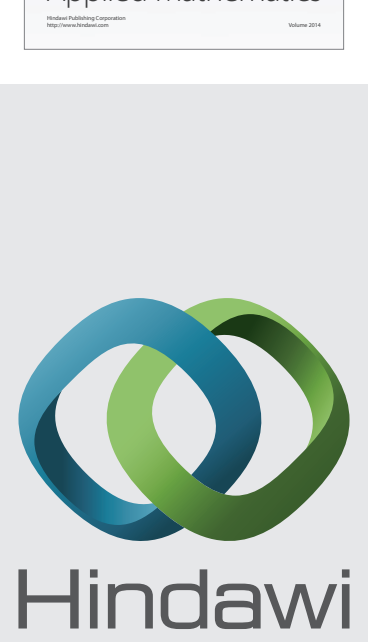

Submit your manuscripts at http://www.hindawi.com
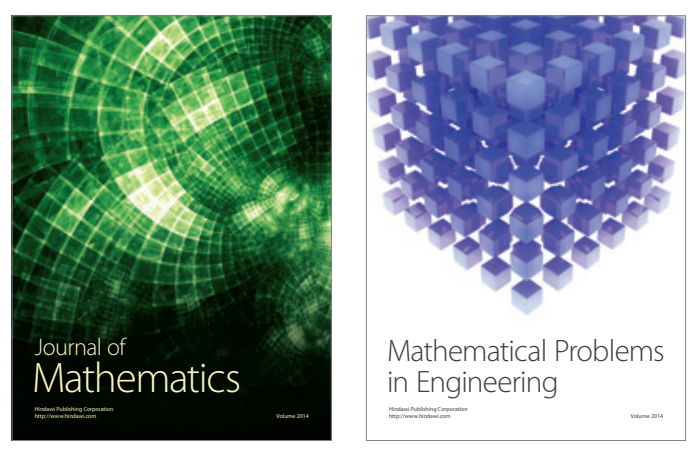

Mathematical Problems in Engineering
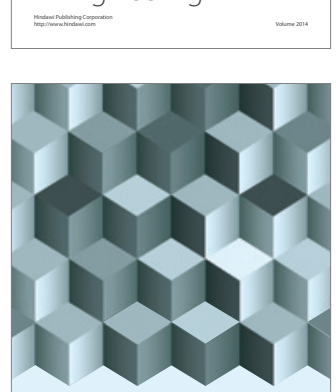

Journal of

Function Spaces
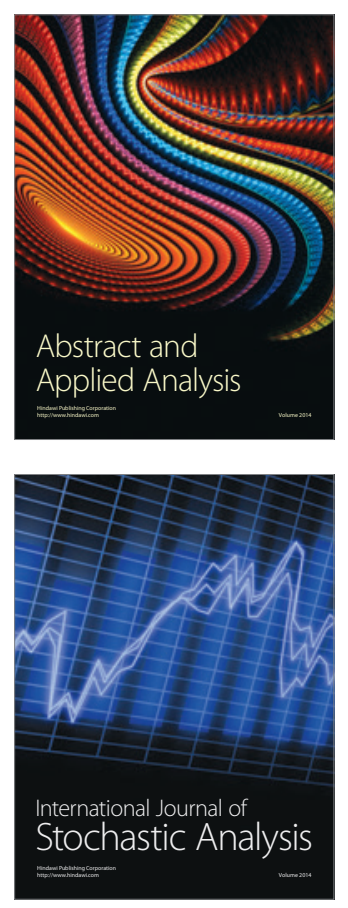

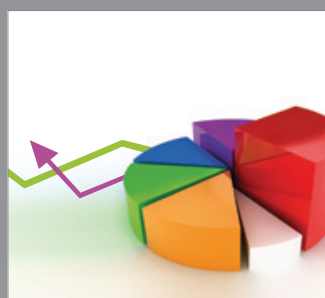

ournal of

Probability and Statistics

Promensencen
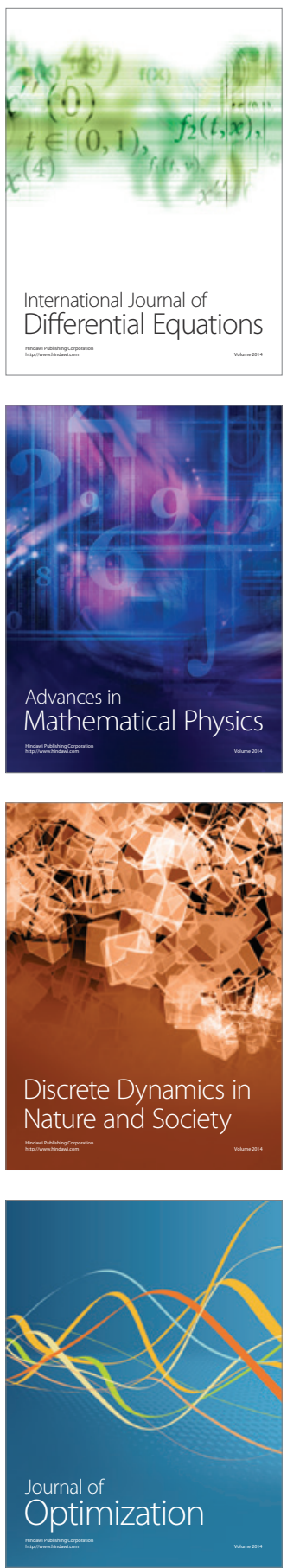